\title{
Lower Limb Refractory Ulcers and Postoperative Nonhealing Wound: Review of the Literature
}

\author{
Shiva S Jha
}

\begin{abstract}
Lower limb ulcers including nonhealing postoperative wounds are challenging problems, especially to orthopedic surgeons. Such ulcers are recognized complications of rheumatoid arthritis but the scenario has changed for the better since the advent of aggressive disease-modifying antirheumatic drugs (DMARDs). Out of different types of lower limb ulcerations including venous, arterial, lymphatic, malignancies, infection, medication induced and inflammatory, autoimmune inflammatory conditions need to be delineated. They can be improved, though slowly with DMARDs. The pathogenesis is multifactorial but most prevalent are vasculitis. The various investigative tools for diagnosing the causes of these ulcers include plethysmography, ultrasound, angiography, computed tomography, magnetic resonance imaging, chromosomal analysis, and, most importantly, skin biopsy. Clinical presentation of the patient still remains the supreme disease tracker. Review of the literature is presented to ascertain the evidence for best clinical practice.
\end{abstract}

Keywords: Disease-modifying antirheumatic drugs, Immunomodulators, Immunosupressants, Infection, Limb ulcers, Pain, Rheumatoid arthritis, Vasculitis.

How to cite this article: Jha SS. Lower Limb Refractory Ulcers and Postoperative Nonhealing Wound: Review of the Literature. J Foot Ankle Surg (Asia-Pacific) 2017;4(1):10-13.

Source of support: Nil

Conflict of interest: None

\section{INTRODUCTION}

Lower limb ulcers (Fig. 1) are challenging problems to orthopedic surgeons, especially when postoperative wounds even without any evidence of local sepsis is not healing and anxiety is writ large on surgeons' face in such a situation.

With orthopedic surgeons' increasing awareness of rheumatology, lower extremity nonhealing wounds, including postoperative ulcers, are now better understood. It simultaneously also reflects on clinical experiences of orthopedicians' successful use of antitubercular treatment in nonhealing postoperative wounds. Ponseti's polyarticular rheumatism is also a known clinical entity in which

\section{Director}

Department of Orthopaedics, Harishchandra Hospital, Patna Bihar, India

Corresponding Author: Shiva S Jha, Director, Department of Orthopaedics, Harishchandra Hospital, Patna, Bihar, India Phone: +06123240681, e-mail: drssjha@gmail.com

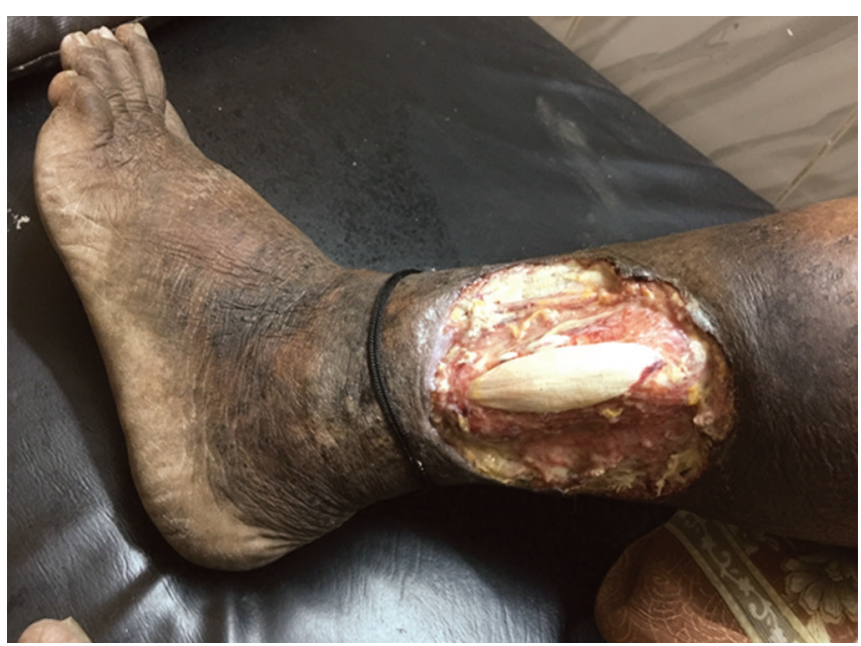

Fig. 1: Giant nontraumatic ulcer

a patient with tuberculosis gets polyarticular involvement, signifying autoimmune factors' initiation even with tubercular infection. Finally, a question definitely remains to be answered - role of antitubercular drugs as immunosuppressants and immunomodulators in wound healing.

Lower extremity ulcers are a recognized complication of rheumatoid arthritis. The involved pathogenesis is multifactorial, ${ }^{1,2}$ and all the following are reported to play a role:

- Vasculitis ${ }^{3}$

- Felty's syndrome (swollen spleen, decreased white blood cell count, and repeated infections $)^{4}$

- Posttraumatic deformity, neuroarthropathy, venous insufficiency

- Arterial disease

Prevalence of approximately 8 to $9 \%$ of leg ulceration in rheumatoid arthritis is reflected in historical cohorts. Seropositive and erosive rheumatoid arthritis is associated with ulceration. Vasculitis and its other extra-articular vasculitic manifestations of rheumatoid arthritis are mainly responsible for these nonhealing ulcers.

\section{Differential Diagnosis}

Factors attributing to various causes are enumerated in Table $1 . .^{5} \mathrm{~A}$ list of whole gamut of other causes not included in Table 1 is detailed in Table 2. Flow chart 1 to proceed after history and clinical examination to differentiate painful and painless ulcers. 


\begin{tabular}{|c|c|}
\hline \multicolumn{2}{|c|}{ Table 1: Causes of leg ulcers } \\
\hline Vascular & Venus \\
\hline & Arterial \\
\hline & Mixed \\
\hline \multirow[t]{3}{*}{ Neuropathic } & Diabetes \\
\hline & Tabes \\
\hline & Syringomyelia \\
\hline \multirow[t]{3}{*}{ Metabolic } & Diabetes \\
\hline & Gout \\
\hline & Prolidase deficiency \\
\hline \multirow[t]{2}{*}{ Hematological } & Sickle cell disease \\
\hline & Cryoglobulinemia \\
\hline \multirow[t]{3}{*}{ Trauma } & Pressure \\
\hline & Injury \\
\hline & Burns \\
\hline \multirow[t]{2}{*}{ Tumors } & Basal cell carcinoma \\
\hline & Squamous cell carcinoma \\
\hline \multirow[t]{3}{*}{ Infection } & Bacterial \\
\hline & Fungal \\
\hline & Protozoal \\
\hline \multirow[t]{2}{*}{ Panniculitis } & Necrobiosis lipoidica \\
\hline & Fat necrosis \\
\hline Pyoderma & Gangrenosum \\
\hline Special & Hypertensive ulcer/obesity \\
\hline
\end{tabular}

\section{Investigation}

Diabetes venous insufficiency and vascular disease must be looked for. Biopsy of the ulcers may be inconclusive in some, but evidence of vasculitis can be delineated. Cholesterol emboli syndrome unusually can also be visible. ${ }^{6}$ Leukocytoclastic vasculitis in tissue adjacent to the ulcer border with an area of intact epidermis can be visualized in positive cases. Common sites for vasculitis are foot (malleolar, dorsal foot, plantar foot) and calf regions. Chromosome analysis, antiphospholipid profile, lupus anticoagulant titers, antiphospholipid antibodies [anti-cardiolipin immunoglobulin A ( $\operatorname{Ig} \mathrm{A})$ antibody and beta-2 glycoprotein $1 \mathrm{IgA}$ ] should be routinely investigated. Genetic prothrombotic study should also be performed. Factor V Leiden mutation, MTHFR C677T PAI-1 mutation details can be part of this genetic observation.

\section{Treatment}

Nonbiologic DMARD, biologic antitumor necrosis factoralpha (anti-TNF alpha) agents are used, but evidence suggests that rheumatoid arthritis-associated ulcers benefit from addition of anti-TNF alpha agents with improved outcome.

Final statistics shows that ulcers remain an important clinical problem (only 30\% of cases had healing in approximately more than 2 months follow-up) although ulcer prevalence has improved with the use of above treatment. In these autoimmune ulcers, it must again be

Physical or
chemical
injury

Table 2: Other causes of limb ulcers

Malignancy Sarcoma, lymphoma, squamous cell carcinoma, basal cell carcinoma, metastatic cancer, Kaposi's and pseudo-Kaposi's sarcoma, cutaneous T-cell and B-cell lymphoma, Hodgkin's disease

Drug induced Steroid ulcus (intralesional injection), vaccination ulcer (Bacillus Calmette-Guérin), halogens, ergotamine, methotrexate, hydroxyurea, paravasal injection of cytostatic and other drugs, granulocyte colony-stimulating factor

Ulcerating Pseudoepitheliomatous hyperplasia, skin diseases epithelioma, pyoderma gangrenosum, pemphigoid, panniculitis, periarteritis nodosa, erythema induratum, Behcet's disease, cutaneous discoid and systemic lupus erythematosus, scleroderma, lichen planus, keratosis actinica, contact dermatitis, fat necrosis, or pancreatic fat necrosis

Autoimmune Dermatitis, lupus, rheumatoid arthritis, vessel: small-vessel leukocytoclastic vasculitis, microscopic polyangiitis, Wegener's granulomatosis, allergic granulomatosis (ChurgStrauss), Henoch-Schonlein purpura, essential cryoglobulinemic vasculitis, erythema induratum Bazin, livedo reticularis, livedo vasculitis and Sneddon syndrome, polyarteritis nodosa, Kawasaki disease

Metabolic Diabetes mellitus, necrobiosis lipoidica, porphyria cutanea tarda, gout, calciphylaxis, calcinosis cutis, homocysteinuria, prolidase deficiency, hyperoxaluria

Hematologic Thalassemia, hereditary spherocytosis,

disorders glucose-6-phosphate dehydrogenase deficiency, essential thrombocythemia, thrombotic thrombocytopenic purpura, granulocytopenia, polycythemia, leukemia, Waldenstrom's disease, multiple myeloma, cryofibrinogenemia, purpura, hyperglobulinemia, cold agglutinins

Clotting Factor V Leiden, lupus anticoagulant, disorders antiphospholipid syndrome, disturbed fibrinolysis, factor XIII deficiency, antithrombin III deficiency, protein $\mathrm{C}$ or $\mathrm{S}$ deficiency, Marcoumar necrosis, large hematoma, purpura fulminans, diffuse intravasal coagulation

emphasized that pathologic features of vasculitis are not always evident of tissue biopsy. All these patients with ulcers in rheumatoid arthritis had radiographic evidence of erosive disease and $63 \%$ were seropositive, suggesting definite correlation of these ulcers with extra-articular rheumatoid disease.

Center for wound healing recommends to adopt a multidisciplinary approach in the management of complex wounds including comprehensive evaluation for venous and arterial disease and aggressive management 
Flow chart 1: Differential diagnosis for lower limb ulceration

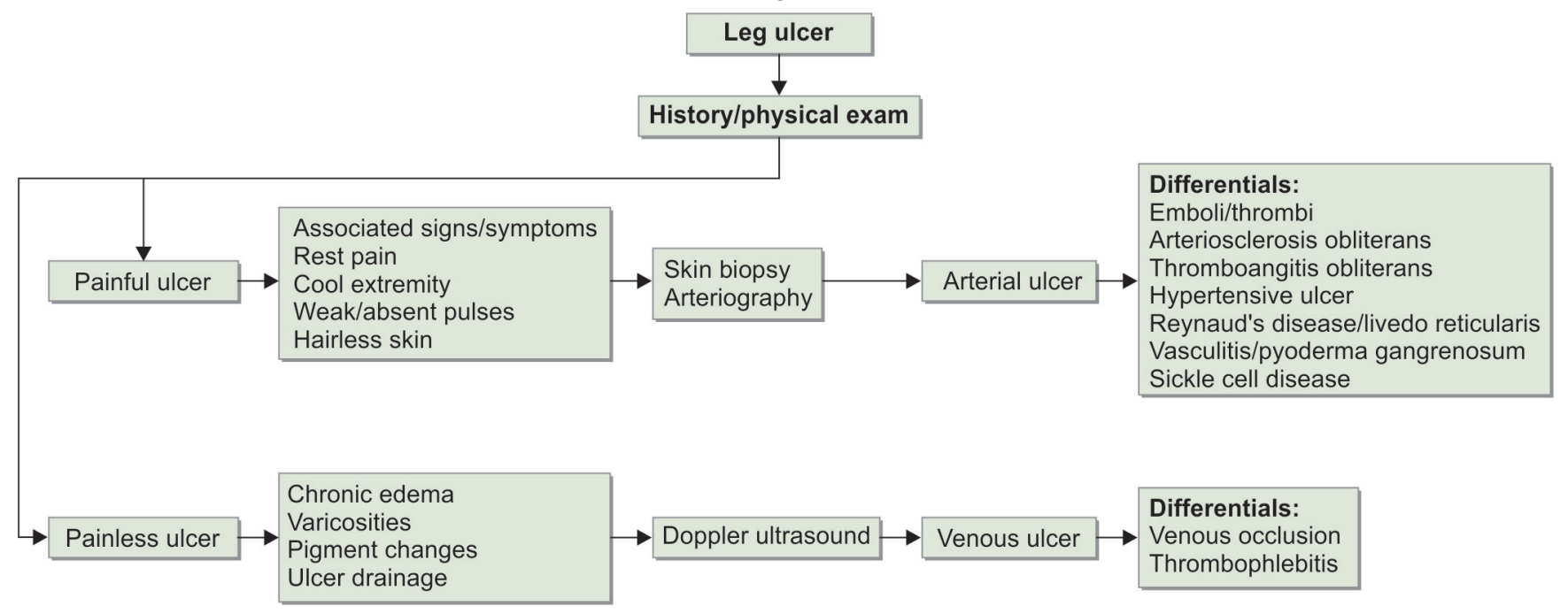

of diabetes, if present. ${ }^{2,3}$ Even in well-controlled diabetics, the wounds refuse to heal; hence, the ulcers are not thought to be due to diabetes.

Other autoimmune diseases like antiphospholipid antibodies, other prothrombotic states, scleroderma ulcers can be prevalent. The endocrinopathy associated with Klinefelter's syndrome has recognized complication of developing lower extremity ulceration, so should be considered as nonhealing lower limb ulcer. Testosterone level and its administration are important in evaluation and its management.

Vasculitis needs special mention: Vasculitis is a term used to describe a group of uncommon diseases which produce inflammatory changes and necrosis in the blood vessel walls. Loss of integrity leads to bleeding, and compromise of the lumen leads to tissue ischemia and necrosis Langford. 7

The outcome of this inflammation depends on the size, type, and location of the vessels involved. Inflammation can affect the aorta and its branches, or may affect medium-sized arteries through to small arteries, venules, and arterioles Scott. ${ }^{8}$ It is small vessel vasculitis that is most commonly associated with cutaneous changes, including nail fold infarct and potentially leg ulceration.

Vasculitis may occur as a primary process or may be secondary to another underlying disease, e.g., rheumatoid arthritis, lupus, or Sjögren's syndrome. Other general features, such as fever, weight loss, and anorexia may accompany widespread inflammation. Therefore, patients often feel systemically unwell.

\section{Diagnosis of Vasculitis}

The diagnosis of vasculitis is often very difficult. If it occurs in combination with another illness, such as rheumatoid arthritis, lupus, or Sjogren's syndrome, then it may be suspected if new or worsened symptoms appear, e.g., weight loss, fever, fatigue, palpable purpura, or nail fold infarcts. A mononeuritis multiplex (i.e., asymmetric neuropathy) is often suggestive of vasculitis in the absence of diabetes Hellmann. ${ }^{9}$

In addition to detailed history and examination, laboratory tests help identify the existence and type of vasculitis and are also useful in identifying any infective influence on the condition. These are often carried out through a specialist unit and are not always available via general community services.

\section{Immunosuppressive Therapies}

Treatment will depend on the type, nature, and severity of the vasculitis. In proven serious vasculitic conditions, powerful therapy is indicated. In initial stages, highdose oral steroid is generally initiated and may be accompanied by 2 to 6 weekly pulses of intravenous cyclophosphamide, an immunosuppressant drug used under specialist direction. It is important not to ignore the potential side effects of these agents. Complications of steroid therapy are well documented Griffiths. ${ }^{10}$ Careful monitoring of opportunistic infection, bone marrow suppression, and hemorrhagic cystitis is necessary. There needs to be awareness of the potential for the subsequent development of bladder tumor and increased risk of other neoplasia Brzeski. ${ }^{11}$ Mortality data suggest that while early deaths in vasculitis are the result of the active disease, late deaths may be caused by the complications of therapy Langford. ${ }^{7}$

The goal of initial treatment is to induce remission of the disease. Once this has been accomplished, the drug dose is lowered to reduce side effects. Low-dose oral steroid, azathioprine, and methotrexate have been used in less severe forms of vasculitis and as maintenance therapy after remission has been induced. 


\section{REFERENCES}

1. Cawley MI. Vasculitis and ulceration in rheumatic diseases of the foot. Baillieres Clin Rheumatol 1987 Aug;1(2): 315-333.

2. Charabaty S, Shanmugam V. A 65-year-old man with longstanding seropositive rheumatoid arthritis and lower extremity ulceration. Arthritis Rheum 2009 Sep;61(9):1275-1280.

3. Nishikawa JA. Are leg ulcers in rheumatoid arthritis due to vasculitis? Eur J Rheumatol Inflamm 1983;6(3):288-290.

4. Oien RF, Håkansson A, Hansen BU. Leg ulcers in patients with rheumatoid arthritis - a prospective study of aetiology, wound healing and pain reduction after pinch grafting. Rheumatology (Oxford) 2001 Jul;40(7):816-820.

5. Sarkar PK, Ballantyne S. Management of leg ulcers. Postgrad Med J 2000 Nov;76(901):674-682.
6. Pun YL, Barraclough DR, Muirden KD. Leg ulcers in rheumatoid arthritis. Med J Aust 1990 Nov;153(10):585-587.

7. Langford CA. Chronic immunosuppressive therapy for systemic vasculitis. Curr Opin Rheumatol 9(1):41-47.

8. Scott G. Vasculitis. In: Butler R, Jayson M, eds. Collected Reports on the Rheumatic Diseases. Arthritis and Rheumatisim Council for Research, Chesterfield: 105-108.

9. Hellmann DB. Practical problems In: Klippel J, Dieppe P (eds). Practical Rheumatology. Times Mirror Publishing Group, London: 408-410

10. Griffiths H, Jordan S. Corticosteroid: implication for practice. Nurs Stand 17(12):43-45.

11. Brzeski M. Oral cyclophosphamide. In: Capell H, Madock R, McInnes I (eds). Practical Prescribing Guidelines in Rheumatoid Arthritis. Martin Dunitz, London: 155-164. 